\title{
Automorphism Groups of Schur Rings
}

\author{
Brent Kerby
}

October 19, 2018

\author{
Brigham Young University, Department of Mathematics, Provo, UT 84604
}

\begin{abstract}
In 1993, Muzychuk [18] showed that the rational Schur rings over a cyclic group $Z_{n}$ are in one-to-one correspondence with sublattices of the divisor lattice of $n$, or equivalently, with sublattices of the lattice of subgroups of $Z_{n}$. This can easily be extended to show that for any finite group $G$, sublattices of the lattice of characteristic subgroups of $G$ give rise to rational Schur rings over $G$ in a natural way. Our main result is that any finite group may be represented as the (algebraic) automorphism group of such a rational Schur ring over an abelian $p$-group, for any odd prime $p$. In contrast, we show that over a cyclic group the automorphism group of any Schur ring is abelian. We also prove a converse to the well-known result of Muzychuk [19] that two Schur rings over a cyclic group are isomorphic if and only if they coincide; namely, we show that over a group which is not cyclic, there always exist distinct isomorphic Schur rings.
\end{abstract}

Schur rings (or S-rings), first developed by I. Schur in [20] in 1933 as a tool for investigating permutation groups, have subsequently found several applications in graph theory and algebraic combinatorics. For a survey of the recent developments in S-rings and their applications, see [17]. All of the definitions and elementary results that we need will be reviewed in \$1.

A great deal of effort has been focused on obtaining a classification of S-rings over cyclic groups, which was achieved in [13, 14]. After the successful classification of S-rings over cyclic groups, a logical next step has been to seek classifications for S-rings over broader families of groups. This, however, appears to be a very difficult problem even in the case, for instance, of abelian $p$-groups. One approach is to restrict attention to special types of S-rings, in particular rational S-rings, in the hope that understanding these may shed light on the general problem. In $\$ 2$ we describe a general construction which, for each sublattice of the lattice of characteristic subgroups of a group $G$, produces a corresponding rational S-ring over $G$. This construction generalizes the one in [18] used to classify rational S-rings over cyclic groups. In $\$ 3$ we review the structure of the lattice of characteristic subgroups over abelian $p$-groups. This enables us to explicitly describe the construction of many rational S-rings over such groups, which we will need for our main result in $\$ 4$. 
We consider the following question: Which groups can occur as the automorphism group of an S-ring? It is clear that the automorphism group of a full group ring $\mathbb{C} G$ (as an S-ring) is isomorphic to the automorphism group of $G$. Thus, any group which may be represented as the automorphism group of a group may also be represented as the automorphism group of an S-ring in a rather trivial way. It is known, however, that there are finite groups (for instance, $Z_{3}$ ) which are not isomorphic to the automorphism group of any group (see, e.g., [22], or [12, Theorem 3.6]). Nevertheless, every finite group occurs as the automorphism group of some S-ring, as our main result shows:

Theorem 4.1 (Main Theorem). Let $p$ be an odd prime. Every finite group can be represented as the automorphism group of a rational S-ring over an abelian p-group.

At the core of the proof is an application of a theorem of Birkhoff that any finite group can be represented as the automorphism group of a finite distributive lattice. In \$5 we use the Leung-Man classification of S-rings over cyclic groups to prove the following theorem, which shows that it is impossible to strengthen our main result by restricting to S-rings over cyclic groups:

Theorem 5.5. If $S$ is an $S$-ring over a cyclic group $Z_{n}$, then $\operatorname{Aut}(S)$ is abelian.

A remarkable theorem of Muzychuk states that over a cyclic group, two S-rings are isomorphic if and only if they coincide [19]. This fact plays a key role in several applications, including our proof of Theorem 5.5. In $₫ 6$, we prove a converse to this result, as follows:

Theorem 6.3. Let $G$ be a finite group which is not cyclic. Then there exist distinct Cayley-isomorphic S-rings $S_{1}$ and $S_{2}$ over $G$.

Finally, in $\$ 7$ we mention some interesting examples of rational S-rings over abelian $p$-groups which cannot be constructed using the method of 92 . We do not yet entirely understand how to generalize these examples, but doing so may be a starting point for approaching the general problem of classifying rational S-rings over abelian $p$-groups, a problem which we believe shows some promise of being tractable.

Note: Most of the results in this paper were included as part of the author's master's thesis [12, under the supervision of Stephen P. Humphries.

\section{Basic definitions}

Let $G$ be a finite group and $F$ a field. If $C$ is a subset of $G$, then we define $\bar{C}$ to be the element $\sum_{g \in C} g$ in the group algebra $F G$, and we call $\bar{C}$ a simple quantity of $F G$. Given a subset $C \subseteq G$ and an integer $m$, we define $C^{(m)}=\left\{g^{m}: g \in C\right\}$. For any $x \in F G$, where $x=\sum_{g \in G} a_{g} g$, we define $x^{(m)}=\sum_{g \in G} a_{g} g^{m}$. Given a subset $X \subseteq F G$, we denote the subspace spanned by $X$ by $F X$. If $\mathcal{L}$ is a collection of subsets of $G$, we let $\overline{\mathcal{L}}$ denote the set $\{\bar{C}: C \in \mathcal{L}\}$. 
A subalgebra $S$ of the group algebra $F G$ is called a $S$ chur ring (or $S$-ring) over $G$ if there are disjoint nonempty subsets $T_{1}, \ldots, T_{n}$ of $G$ such that $\bar{T}_{1}, \ldots, \bar{T}_{n}$ form a basis for $S$, with the following properties,

(i) For every $i$ there is some $j$ such that $T_{i}^{(-1)}=T_{j}$.

(ii) $T_{1}=\{1\}$, and $G=T_{1} \cup T_{2} \cup \cdots \cup T_{n}$.

The sets $T_{1}, \ldots, T_{n}$ are called basic sets of $S$ and are said to form a Schur partition of $G$. The corresponding $\bar{T}_{1}, \ldots, \bar{T}_{n}$ are called the basic quantities of $S$. If $S$ satisfies condition (i), but perhaps not (ii), then $S$ is called a pseudo $S$-ring (or $P S$-ring). The two sets $\{1\}, G \backslash\{1\}$ always form a Schur partition; the corresponding S-ring is called the trivial S-ring over $G$.

Given $x, y \in F G$, where $x=\sum_{g \in G} a_{g} g, y=\sum_{g \in G} b_{g} g$, their Hadamard product is defined by

$$
x \circ y=\sum_{g \in G} a_{g} b_{g} g .
$$

There is a well-known purely algebraic description of S-rings and PS-rings in terms of closure under the Hadamard product, avoiding reference to the combinatorial notion of basic sets (for proofs, see [18, Proposition 3.1] and [19, Lemma 1.3], or [12, Theorem 1.7, Corollary 1.8]):

Theorem 1.1. Let $A$ be a subalgebra of $F G$. Then $A$ is a $P S$-ring if and only if $A$ is closed under $\circ$ and ${ }^{(-1)}$.

Corollary 1.2. Let $A$ be a subalgebra of $F G$. Then $A$ is an $S$-ring if and only if $A$ is closed under $\circ$ and ${ }^{(-1)}$ and contains 1 and $\bar{G}$.

Definition 1.3. Let $S_{1}$ and $S_{2}$ be S-rings over groups $G_{1}$ and $G_{2}$ respectively. An algebra isomorphism $\phi: S_{1} \rightarrow S_{2}$ is called an isomorphism of S-rings if $\phi$ maps basic quantities to basic quantities, i.e., if for every basic set $C$ of $S_{1}$ there is some basic set $D$ of $S_{2}$ such that $\phi(\bar{C})=\bar{D}$.

The following well-known result gives a purely algebraic characterization of S-ring isomorphisms (an elementary proof may be found in [12, Theorem 3.4]):

Theorem 1.4. Let $S_{1}$ and $S_{2}$ be $S$-rings over groups $G_{1}$ and $G_{2}$ respectively. Then an $F$-algebra isomorphism $\phi: S_{1} \rightarrow S_{2}$ is an $S$-ring isomorphism if and only if $\phi$ respects the Hadamard product, i.e., if and only if $\phi(x \circ y)=\phi(x) \circ \phi(y)$ for all $x, y \in S_{1}$.

Elsewhere in the literature, for clarity, an isomorphism of S-rings is sometimes called an algebraic isomorphism, in contrast to the notion of a combinatorial isomorphism of S-rings, which, properly speaking, is an isomorphism of the association schemes corresponding to the S-rings.

If $\bar{C}$ is any simple quantity contained in an S-ring $S$, then we say that $C$ is an $S$-set. The following is also well-known (For a proof, see [12, Theorem 3.7, Corollary $6.8])$ : 
Theorem 1.5. Let $\phi: S_{1} \rightarrow S_{2}$ be an isomorphism of $S$-rings over groups $G_{1}$ and $G_{2}$ respectively. Let $C \subseteq G_{1}$ be an $S_{1}$-set. Then $\phi(\bar{C})=\bar{D}$ for some $S_{2}$-set $D \subseteq G_{2}$, and we write $\phi(C)=D$. Moreover,

(i) $|C|=|D|$, and

(ii) If $C$ is a subgroup then $D$ is also a subgroup.

An isomorphism from an S-ring onto itself is called an automorphism. The set of automorphisms of an S-ring $S$ is denoted $\operatorname{Aut}(S)$. Every group automorphism in $\operatorname{Aut}(G)$ naturally induces an S-ring isomorphism of $S$ (onto a possibly distinct Sring); such an isomorphism is called a Cayley isomorphism, and the two S-rings are called Cayley-isomorphic.

It is possible to consider S-rings where the coefficient field $F$ is replaced by an arbitrary ring $R$ (with unity). The collection of Schur partitions is affected only by the characteristic of $R$; so whether $R$ is $\mathbb{Z}, \mathbb{Q}, \mathbb{C}$, or any other ring of characteristic 0 makes no difference, as far as the collection of Schur partitions is concerned. Nevertheless, Theorem 1.1 and Corollary 1.2 both become false over any commutative ring $R$ which is not a field; for this reason, we will always take our coefficient ring to be a field $F$. Over rings of nonzero characteristic, the collection of Schur partitions includes all those associated with zero characteristic in addition possibly to others. (For proof of these statements, see [12, Example 1.10, Theorem 1.12].) It is possible for the isomorphism class of the automorphism group $\operatorname{Aut}(S)$ to change depending on the characteristic of the coefficient field $F$ (see Example 5.9 below). Throughout the literature on S-rings, most authors assume a coefficient ring of characteristic zero. We will also need this assumption in $\$ 5$ when we show that the automorphism group of an S-ring over a cyclic group is abelian; indeed, this theorem becomes false over any ring of nonzero characteristic, as Example 5.9 will show.

\section{Central and rational S-rings}

Definition 2.1. A PS-ring $S$ over a group $G$ is central if $S \subseteq Z(F G)$, i.e., $S$ is contained in the center of the group algebra.

Remark. This is equivalent to requiring that every basic set $T_{i}$ be a union of conjugacy classes of $G$.

Of course, over an abelian group every S-ring is central. We are primarily interested in a special class of central S-rings known as rational S-rings:

Definition 2.2. A PS-ring $S$ over a group $G$ is rational if for every $x \in S$ and $\phi \in \operatorname{Aut}(G)$, we have $\phi(x)=x$.

Remark. This is equivalent to requiring that every basic set $T_{i}$ be a union of automorphism classes of $G$, where the automorphism classes of $G$ are the orbits of $\operatorname{Aut}(G)$ acting on $G$ in the natural way. 
The following provides a method for constructing many interesting central and rational S-rings:

Theorem 2.3. Let $G$ be any finite group, and let $\mathcal{L}$ be any sublattice of the lattice of normal subgroups of $G$. Then the vector space $F \overline{\mathcal{L}}$ is a central PS-ring over $G$ with the following properties, for all $H, K \in \mathcal{L}$ :

(i) $\bar{H}^{(-1)}=\bar{H}$

(ii) $\bar{H} \circ \bar{K}=\overline{H \cap K}$

(iii) $\bar{H} \bar{K}=|H \cap K| \overline{H K}$

(iv) $F \overline{\mathcal{L}}$ is an $S$-ring if and only if $1, G \in \mathcal{L}$.

(v) $F \overline{\mathcal{L}}$ is rational if and only if $\mathcal{L}$ consists entirely of characteristic subgroups.

Proof. (i) is clear since $H$, as a subgroup, is closed under inverses. (ii) is immediate from the definition of the Hadamard product. (iii) is clear since, by elementary group theory, every element of $H K$ can be written in $|H \cap K|$ ways as a product of an element in $H$ with an element in $K$. Now, since the subgroups $H$ and $K$ are normal, $H K$ is also a subgroup of $G$; since $\mathcal{L}$ is a lattice, we have $H \cap K, H K \in \mathcal{L}$. Thus, (i)(iii) show that $F \overline{\mathcal{L}}$ is closed under ${ }^{(-1)}$, multiplication, and the Hadamard product, so by Theorem 1.1, $F \overline{\mathcal{L}}$ is a PS-ring. Since each subgroup $H$ is normal, we have $g \bar{H} g^{-1}=\overline{g H g^{-1}}=\bar{H}$ for all $g \in G$ and $H \in \mathcal{L}$, so that $\bar{H} \in Z(F G)$. It follows that $F \overline{\mathcal{L}}$ is a central PS-ring.

Now, if $1, G \in \mathcal{L}$ then $1, \bar{G} \in F \overline{\mathcal{L}}$, so by Corollary $1.2, F \overline{\mathcal{L}}$ is an S-ring. Suppose conversely that $F \overline{\mathcal{L}}$ is an S-ring. Let $L=\bigcap_{H \in \mathcal{L}} H$, so that $L \in \mathcal{L}$. If $|L|>1$, then since $L \subseteq H$ for every spanning element $\bar{H}$ of $F \overline{\mathcal{L}}$, it follows that every element of $F \overline{\mathcal{L}}$ with a non-zero coefficient of $1 \in G$ also has other non-zero coefficients (namely, the other elements of $L$ have non-zero coefficients), so that $1 \notin F \overline{\mathcal{L}}$, contrary to the assumption that $F \overline{\mathcal{L}}$ is an S-ring. So we must have $L=1$, hence $1 \in \mathcal{L}$, as desired. Now define $M=\prod_{H \in \mathcal{L}} H$. If $M \neq G$, then since $H \subseteq M$ for every spanning element $\bar{H}$ of $F \overline{\mathcal{L}}$, it follows that the nonzero coefficients of every element $x \in F \overline{\mathcal{L}}$ are contained in $M$, so that $\bar{G} \notin F \overline{\mathcal{L}}$, again contradicting that $F \overline{\mathcal{L}}$ is an S-ring. So $G \in \mathcal{L}$ as desired.

Finally, $F \overline{\mathcal{L}}$ is rational if and only if $\phi(\bar{H})=\bar{H}$ for every $\phi \in \operatorname{Aut}(G)$ and each spanning element $\bar{H}$ of $F \overline{\mathcal{L}}$; this holds if and only if $\phi(H)=H$ for each $H \in \mathcal{L}$, i.e. if and only if each $H \in \mathcal{L}$ is characteristic.

If $G$ is a cyclic group, then every subgroup of $G$ is characteristic; consequently, the construction of Theorem 2.3 produces only rational S-rings. In this context, we may state the main theorem of [18] as follows:

Theorem 2.4. (Muzychuk)Every rational S-ring over a finite cyclic group may be constructed as in Theorem [2.3. 
There are rational S-rings over abelian $p$-groups which cannot be constructed as in Theorem 2.3 (see Example 7.1). However, there are other types of groups for which Theorem 2.3 produces the complete set of rational S-rings. We mention an example, whose proof can be found in [12, Theorem 2.6]:

Theorem 2.5. Every rational S-ring over a finite dihedral group may be constructed as in Theorem 2.3.

\section{Characteristic subgroups of abelian $p$-groups}

In this section, we review the description of the automorphism classes and characteristic subgroups of finite abelian p-groups. This topic was considered in 1905 and 1920 by G. A. Miller [15, 16] and again, independently, in 1934 by Baer, who considered the more general case of periodic abelian groups [1], and finally in 1935 by Birkhoff [2]. The historical nature of these early works is such that they are not easy reading; the present author confesses that in many places the statements made in them, and particularly the proofs, do not always seem clear. A more recent treatment may be found in [11] (or [12]), which includes proofs of all the results below as well as a detailed description of the exceptional case $p=2$.

Throughout this section, $G$ will denote a finite abelian $p$-group:

$$
G=Z_{p^{\lambda_{1}}} \times Z_{p^{\lambda_{2}}} \times \cdots \times Z_{p^{\lambda_{n}}},
$$

where $\lambda_{1} \leq \lambda_{2} \leq \cdots \leq \lambda_{n}$. We define $\lambda(G)$ to be the tuple $\left(\lambda_{1}, \ldots, \lambda_{n}\right)$. For convenience, we will often use the convention $\lambda_{0}=0$. As we will be working extensively with such tuples of integers, it will be useful to introduce some notation for dealing with them:

Definition 3.1. Given tuples $\mathbf{a}=\left(a_{1}, \ldots, a_{n}\right)$ and $\mathbf{b}=\left(b_{1}, \ldots, b_{n}\right)$ with integer entries, define

$$
\begin{aligned}
& \mathbf{a} \leq \mathbf{b} \text { if } a_{i} \leq b_{i} \text { for all } i \in\{1, \ldots, n\} \\
& \mathbf{a} \wedge \mathbf{b}=\left(\min \left\{a_{1}, b_{1}\right\}, \min \left\{a_{2}, b_{2}\right\}, \ldots, \min \left\{a_{n}, b_{n}\right\}\right) \\
& \mathbf{a} \vee \mathbf{b}=\left(\max \left\{a_{1}, b_{1}\right\}, \max \left\{a_{2}, b_{2}\right\}, \ldots, \max \left\{a_{n}, b_{n}\right\}\right)
\end{aligned}
$$

Define $\Lambda(G)$ to be the set of tuples

$$
\Lambda(G)=\{\mathbf{a}: \mathbf{0} \leq \mathbf{a} \leq \lambda(G)\} .
$$

It is evident that $\Lambda(G)$, under the partial order $\leq$, forms a finite lattice in which $\wedge$ and $\vee$ are the greatest lower bound and least upper bound respectively.

Given a tuple $\mathbf{a} \in \Lambda(G)$, we define $T(\mathbf{a})$ to be the set of elements $g \in G$ for which the $i$ th component of $g$ has order $p^{a_{i}}$ :

$$
T(\mathbf{a})=\left\{\left(g_{1}, g_{2}, \ldots, g_{n}\right) \in G:\left|g_{i}\right|=p^{a_{i}} \text { for all } i=1, \ldots, n\right\} .
$$

Note that the sets $T(\mathbf{a})$ partition the group $G$. If $g \in T(\mathbf{a})$, we say that the type of $g$ is $T(\mathbf{a})$.

The following is straightforward to prove: 
Lemma 3.2. If two elements have the same type, then they are in the same automorphism class.

Definition 3.3. Given a type $T(\mathbf{a})$, the automorphism class of $G$ containing $T(\mathbf{a})$ is denoted $O(\mathbf{a})$.

Definition 3.4. A tuple $\mathbf{a}=\left(a_{1}, \ldots, a_{n}\right)$ and its corresponding type $T(\mathbf{a})$ are called canonical if for all $i \in\{1, \ldots, n-1\}$,

(i) $a_{i} \leq a_{i+1}$ and

(ii) $a_{i+1}-a_{i} \leq \lambda_{i+1}-\lambda_{i}$.

The definition of "canonical" is justified by the following theorem.

Theorem 3.5. Every automorphism class contains a unique canonical type.

Example 3.6. Let $G=Z_{2} \times Z_{8}=Z_{2} \times Z_{2^{3}}=\langle s\rangle \times\langle t\rangle$. Then there are 6 automorphism classes of $G$, namely:

$$
\begin{aligned}
& O(0,0)=T(0,0)=\{1\} \\
& O(0,1)=T(0,1)=\left\{t^{4}\right\} \\
& O(0,2)=T(0,2)=\left\{t^{2}, t^{6}\right\} \\
& O(1,1)=T(1,1) \cup T(1,0)=\left\{s, s t^{4}\right\} \\
& O(1,2)=T(1,2)=\left\{s t^{2}, s t^{6}\right\} \\
& O(1,3)=T(1,3) \cup T(0,3)=\left\{t, s t, t^{3}, s t^{3}, t^{5}, s t^{5}, t^{7}, s t^{7}\right\} .
\end{aligned}
$$

Having established a sufficiently detailed description of the automorphism classes for our purposes, we now turn to the characteristic subgroups. We let Char $(G)$ denote the lattice of characteristic subgroups of $G$.

Definition 3.7. Given a tuple $\mathbf{a} \in \Lambda(G)$, we define the subgroup $R(\mathbf{a})=\underset{\mathbf{b} \leq \mathbf{a}}{\cup} T(\mathbf{b})$ and call $R(\mathbf{a})$ the regular subgroup below $\mathbf{a}$.

Remark. We use the term "regular", following Baer ([1]). But this concept of regular should not be confused with the notion of a regular permutation group, nor of a regular $p$-group.

Theorem 3.8. $R(\mathbf{a})$ is a characteristic subgroup if and only if a is canonical.

The following is easily verified by direct calculation:

Theorem 3.9. For any $\mathbf{a}, \mathbf{b} \in \Lambda(G)$,

(i) $R(\mathbf{a}) \cap R(\mathbf{b})=R(\mathbf{a} \wedge \mathbf{b})$

(ii) $\langle R(\mathbf{a}), R(\mathbf{b})\rangle=R(\mathbf{a} \vee \mathbf{b})$ 
(iii) $|R(\mathbf{a})|=p^{\sum_{i=1}^{n} a_{i}}$

From (i) and (ii), it follows that the regular characteristic subgroups form a sublattice of $\operatorname{Char}(G)$. Using Theorem 3.8 , this then implies that if $\mathbf{a}$ and $\mathbf{b}$ are canonical tuples then so are $\mathbf{a} \wedge \mathbf{b}$ and $\mathbf{a} \vee \mathbf{b}$. (This is also not difficult to verify directly.) Thus the canonical tuples form a sublattice of $\Lambda(G)$; this sublattice will be denoted by $\mathcal{C}(G)$.

The following theorem is of great importance to us:

Theorem 3.10 (Miller-Baer). If $p \neq 2$, then every characteristic subgroup of $G$ is regular.

We then have the following important corollaries:

Corollary 3.11. If $p \neq 2$, then $\operatorname{Char}(G) \cong \mathcal{C}(G)$.

Corollary 3.12 (Miller-Baer-Birkhoff). For any prime $p$, the number of automorphism classes of $G=Z_{p^{\lambda_{1}}} \times Z_{p^{\lambda_{2}}} \times \cdots \times Z_{p^{\lambda_{n}}}$ is

$$
\prod_{i=1}^{n}\left(\lambda_{i}-\lambda_{i-1}+1\right) .
$$

If $p \neq 2$, then this is also the number of characteristic subgroups of $G$.

Proof. We apply Corollary 3.11, observing that the canonical tuples a are precisely those which satisfy $a_{i-1} \leq a_{i} \leq a_{i-1}+\lambda_{i}-\lambda_{i-1}$ for each $i \in\{1, \ldots, n\}$. Thus there are $\lambda_{i}-\lambda_{i-1}+1$ choices for each coordinate $a_{i}$, and the first statement follows. The second follows from the first and Theorems 3.8 and 3.10 .

We define $\mathcal{W}(G)$ to be the subalgebra of elements of $F G$ which are invariant under $\operatorname{Aut}(G)$. Thus, $\mathcal{W}(G)$ is the maximum rational S-ring over $F G$; its basic sets are the automorphism classes of $G$. We then obtain one last corollary:

Corollary 3.13. If $p \neq 2$, then $\overline{\operatorname{Char}(G)}$ is a basis for $\mathcal{W}(G)$.

Proof. Clearly $\overline{\operatorname{Char}(G)} \subseteq \mathcal{W}(G)$. By Theorem 3.5, the S-ring $\mathcal{W}(G)$ is spanned by $\{\overline{O(\mathbf{a})}: \mathbf{a} \in \mathcal{C}(G)\}$. For any canonical tuple $\mathbf{a} \in \mathcal{C}(G)$, we can write

$$
\overline{O(\mathbf{a})}=\overline{R(\mathbf{a})}-\sum_{\substack{\mathbf{b} \in \mathcal{C}(G) \\ \mathbf{b}<\mathbf{a}}} \overline{O(\mathbf{b})},
$$

and, since $\overline{R(\mathbf{a})} \in \overline{\mathrm{Char}(G)}$, it follows by induction that each $\overline{O(\mathbf{a})}$ is in the span of $\overline{\operatorname{Char}(G)}$. Since $\operatorname{dim}(\mathcal{W}(G))=|\operatorname{Char}(G)|$ by Corollary 3.12 , the result follows.

Example 3.14. Let $G=Z_{p} \times Z_{p^{3}}$ for an odd prime $p$. Then the characteristic subgroups of $G$ are shown in Table 1 . 
Table 1: Characteristic subgroups of $G=Z_{p} \times Z_{p^{3}}$ for odd prime $p$

\begin{tabular}{|l|l|}
\hline$H_{1}$ & $\mathrm{R}(0,0)$ \\
\hline$H_{2}$ & $\mathrm{R}(0,1)$ \\
\hline$H_{3}$ & $\mathrm{R}(0,2)$ \\
\hline$H_{4}$ & $\mathrm{R}(1,1)$ \\
\hline$H_{5}$ & $\mathrm{R}(1,2)$ \\
\hline$H_{6}$ & $\mathrm{R}(1,3)$ \\
\hline
\end{tabular}

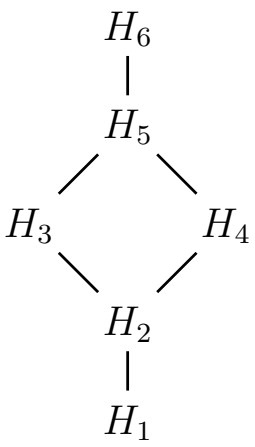

Table 2: Characteristic subgroups of $G=Z_{p} \times Z_{p^{3}} \times Z_{p^{5}}$ for odd prime $p$

\begin{tabular}{|l|l|}
\hline$H_{1}$ & $\mathrm{R}(0,0,0)$ \\
\hline$H_{2}$ & $\mathrm{R}(0,0,1)$ \\
\hline$H_{3}$ & $\mathrm{R}(0,0,2)$ \\
\hline$H_{4}$ & $\mathrm{R}(0,1,1)$ \\
\hline$H_{5}$ & $\mathrm{R}(0,1,2)$ \\
\hline$H_{6}$ & $\mathrm{R}(0,1,3)$ \\
\hline$H_{7}$ & $\mathrm{R}(0,2,2)$ \\
\hline$H_{8}$ & $\mathrm{R}(0,2,3)$ \\
\hline$H_{9}$ & $\mathrm{R}(0,2,4)$ \\
\hline$H_{10}$ & $\mathrm{R}(1,1,1)$ \\
\hline$H_{11}$ & $\mathrm{R}(1,1,2)$ \\
\hline$H_{12}$ & $\mathrm{R}(1,1,3)$ \\
\hline$H_{13}$ & $\mathrm{R}(1,2,2)$ \\
\hline$H_{14}$ & $\mathrm{R}(1,2,3)$ \\
\hline$H_{15}$ & $\mathrm{R}(1,2,4)$ \\
\hline$H_{16}$ & $\mathrm{R}(1,3,3)$ \\
\hline$H_{17}$ & $\mathrm{R}(1,3,4)$ \\
\hline$H_{18}$ & $\mathrm{R}(1,3,5)$ \\
\hline
\end{tabular}

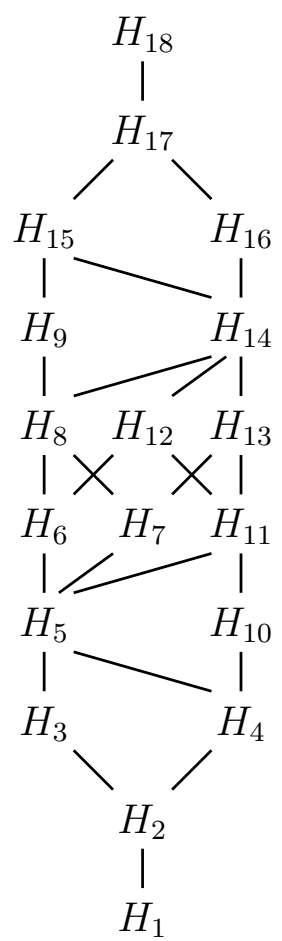


Example 3.15. Let $G=Z_{p} \times Z_{p^{3}} \times Z_{p^{5}}$ for an odd prime $p$. Then the characteristic subgroups of $G$ are shown in Table 2. Note that the sublattice of $\operatorname{Char}(G)$ between $H_{5}=R(0,1,2)$ and $H_{14}=R(1,2,3)$ forms a cube; i.e., this sublattice is isomorphic to the boolean lattice $\mathcal{P}(X)$ of subsets of a set $X$ of cardinality 3 .

The following theorem gives a generalization of preceding two examples which will be important to us in 44

Theorem 3.16. Let $X$ be a (finite) set containing $n$ elements, and let

$$
G=Z_{p} \times Z_{p^{3}} \times \cdots \times \mathbb{Z}_{p^{2 n-1}} .
$$

Then there is an embedding $\psi$ of the boolean lattice $\mathcal{P}(X)$ into Char $(G)$. Further, $\psi$ has the property that for all subsets $Y_{1}, Y_{2}$ of $X,\left|Y_{1}\right|=\left|Y_{2}\right| \Longleftrightarrow\left|\psi\left(Y_{1}\right)\right|=\left|\psi\left(Y_{2}\right)\right|$.

Proof. We will map $\mathcal{P}(X)$ onto the sublattice of Char $(G)$ between $R(0,1,2, \ldots, n-1)$ and $R(1,2,3, \ldots, n)$ in the following way: Write $X=\left\{x_{1}, \ldots, x_{n}\right\}$; then, for $Y \subseteq X$ define $\psi(Y)=R(\mathbf{a})$, where $\mathbf{a}=\left(a_{1}, \ldots, a_{n}\right)$ is given by

$$
a_{i}= \begin{cases}i-1, & \text { if } x_{i} \notin Y \\ i, & \text { if } x_{i} \in Y\end{cases}
$$

Note that $\psi$ is well-defined since each the tuple a, as thus defined, is always canonical, since the difference between two consecutive components is either 0,1 , or 2 . We have

$$
\begin{aligned}
\psi\left(Y_{1} \cap Y_{2}\right) & =R\left(\mathbf{a}\left(Y_{1} \cap Y_{2}\right)\right)=R\left(\mathbf{a}\left(Y_{1}\right) \wedge \mathbf{a}\left(Y_{2}\right)\right) \\
& =R\left(\mathbf{a}\left(Y_{1}\right)\right) \cap R\left(\mathbf{a}\left(Y_{2}\right)\right)=\psi\left(Y_{1}\right) \cap \psi\left(Y_{2}\right)
\end{aligned}
$$

and

$$
\begin{aligned}
\psi\left(Y_{1} \cup Y_{2}\right) & =R\left(\mathbf{a}\left(Y_{1} \cup Y_{2}\right)\right)=R\left(\mathbf{a}\left(Y_{1}\right) \vee \mathbf{a}\left(Y_{2}\right)\right) \\
& =\left\langle R\left(\mathbf{a}\left(Y_{1}\right)\right), R\left(\mathbf{a}\left(Y_{2}\right)\right)\right\rangle=\left\langle\psi\left(Y_{1}\right), \psi\left(Y_{2}\right)\right\rangle,
\end{aligned}
$$

so that $\psi$ is a lattice homomorphism. By construction $\psi$ is injective, so $\psi$ is an embedding. Theorem 3.9(iii) shows that $|\psi(Y)|=p^{\frac{n(n-1)}{2}+|Y|}$, and the last claim follows.

\section{Main Theorem}

We now turn to our main result. In this section, we take all S-rings over a coefficient field $F$ of characteristic 0 .

Theorem 4.1. (Main Theorem) Let $p$ be an odd prime. Every finite group can be represented as the automorphism group of a rational S-ring over an abelian p-group.

The proof relies on three key ideas: 
(1) Every finite group can be represented as the automorphism group of a finite distributive lattice,

(2) Every distributive lattice can be embedded in a boolean lattice, and

(3) The lattice of characteristic subgroups of the group $Z_{p} \times Z_{p^{3}} \times \cdots \times Z_{p^{2 n-1}}$ contains a boolean sublattice of order $2^{n}$.

The proof of the Main Theorem, in outline, goes as follows: Given a group $G$, by (1) there is a distributive lattice with automorphism group isomorphic to $G$. By (2), this lattice may be embedded in a boolean lattice, which by (3) may in turn be embedded as a sublattice of the lattice of characteristic subgroups of an appropriate abelian $p$-group $P$. Finally, using Theorem 2.3 , we construct the rational S-ring over $P$ associated with this lattice and show that the automorphism group of this S-ring is isomorphic to $\mathrm{G}$.

(1) was shown by Birkhoff in [3]; a proof may also be found in [12, §7], [9], or [8] (see also [7]). (2) is a standard result in lattice theory (also first proven by Birkhoff); a proof may be found in [6, Theorem 5.12], [10, 20.1], [5, 11.3], or [12, Theorem 6.6]. We have already shown (3) in Theorem 3.16 above. So all that remains is to verify the last step of our argument. Before doing this, however, we will need to give a more explicit description of the embedding of $(2)$ :

Recall that an element $j$ of a lattice $\mathcal{L}$ is join-reducible if there exist $x<j$ and $y<j$ with $j=x \vee y$. Otherwise $j$ is said to be join-irreducible.

Theorem 4.2 (Birkhoff). Let $\mathcal{L}$ be a finite distributive lattice and let $J \subseteq \mathcal{L}$ be the set of join-irreducible elements. Then the map $\phi(x)=\{j \in J: j \leq x\}$ is a embedding of $\mathcal{L}$ into $\mathcal{P}(J)$.

In what follows, we will need the following fact:

Theorem 4.3. In Theorem 4.2, $|\phi(\alpha(x))|=|\phi(x)|$ for all $\alpha \in \operatorname{Aut}(\mathcal{L})$ and $x \in \mathcal{L}$.

Proof. Since lattice isomorphisms permute the join-irreducible elements among themselves, we have

$$
\begin{aligned}
\phi(\alpha(x)) & =\{j \in J: j \leq \alpha(x)\}=\{\alpha(j) \in J: \alpha(j) \leq \alpha(x)\} \\
& =\{\alpha(j) \in J: j \leq x\}=\alpha(\phi(x)),
\end{aligned}
$$

hence $|\phi(\alpha(x))|=|\alpha(\phi(x))|=|\phi(x)|$.

Now we are able to prove the Main Theorem. Given a finite group $G$, by (1) there is a finite distributive lattice $\mathcal{D}$ with $\operatorname{Aut}(D) \cong G$. By Theorem 4.2, there is an embedding $\phi$ of $\mathcal{D}$ into the boolean lattice $\mathcal{P}(J)$, where $J$ is the set of join-irreducible elements of $\mathcal{D}$. In turn, by Theorem 3.16, there is an embedding $\psi$ of $\mathcal{P}(J)$ into the lattice of characteristic subgroups $\operatorname{Char}(P)$ of an appropriate abelian $p$-group $P$. Let $\mathcal{L}=\psi(\phi(D)) \cup\{1\} \cup\{P\}$. Then by Theorem 2.3, $F \overline{\mathcal{L}}$ is an S-ring over $P$. To prove the theorem, we will show that $\operatorname{Aut}(F \overline{\mathcal{L}}) \cong G$. 
Since every automorphism of $\mathcal{L}$ fixes 1 and $P$ and hence restricts to an automorphism of $\psi(\phi(D))$, it follows that $\operatorname{Aut}(\psi(\phi(D))) \cong \operatorname{Aut}(\mathcal{L})$. So since $D \cong \psi(\phi(D))$, we then have $G \cong \operatorname{Aut}(D) \cong \operatorname{Aut}(\psi(\phi(D))) \cong \operatorname{Aut}(\mathcal{L})$. So it suffices to show $\operatorname{Aut}(\mathcal{L}) \cong \operatorname{Aut}(F \overline{\mathcal{L}})$.

We define a map $\chi: \operatorname{Aut}(\mathcal{L}) \rightarrow \operatorname{Aut}(F \overline{\mathcal{L}})$ by $\chi(\beta): \bar{H} \mapsto \overline{\beta(H)}$ for $H \in \mathcal{L}$. We need to justify that $\chi$ is well-defined, i.e., that $\chi(\beta)$ actually is an S-ring automorphism of $\mathcal{L}$. After that, it will be clear that $\chi$ is an injective homomorphism. Proving the surjectivity of $\chi$ will then complete the proof.

Let $\beta \in \operatorname{Aut}(\mathcal{L})$. Since Corollary 3.13 implies that $\overline{\mathcal{L}}$ is linearly independent and hence forms a basis for $F \overline{\mathcal{L}}$, it is clear that $f=\chi(\beta)$ is a well-defined bijective linear map from $F \overline{\mathcal{L}}$ to itself. To show that $f$ is an S-ring automorphism, we just need to show that it preserves the Hadamard and ordinary products. Applying Theorem 2.3, we have

$$
\begin{aligned}
f(\bar{H} \circ \bar{K}) & =f(\overline{H \cap K})=\overline{\beta(H \cap K)}=\overline{\beta(H) \cap \beta(K)} \\
& =\overline{\beta(H)} \circ \overline{\beta(K)}=f(\bar{H}) \circ f(\bar{K}),
\end{aligned}
$$

so $f$ preserves the Hadamard product. Before we can show $f$ preserves the ordinary product, we first need the result that for all $H \in \mathcal{L},|\beta(H)|=|H|$. For $H=1$ or $H=P$ this is trivially true since in these cases $\beta(H)=H$. For any other $H$, we may write $H=\psi(\phi(x))$ for some $x \in D$. Now, letting $\alpha \in \operatorname{Aut}(D)$ be the composite function $\alpha=\phi^{-1} \psi^{-1} \beta \psi \phi$, we see that showing $|\beta(H)|=|H|$ is equivalent to showing $|\psi(\phi(\alpha(x)))|=|\psi(\phi(x))|$, which by Theorem 3.16 is equivalent to showing $|\phi(\alpha(x))|=$ $|\phi(x)|$, which in turn holds by Theorem 4.3. So we have proven $|\beta(H)|=|H|$. In particular, if $H, K \in \mathcal{L}$, we have

$$
|H \cap K|=|\beta(H \cap K)|=|\beta(H) \cap \beta(K)|,
$$

and from this, by applying Theorem 2.3 , we obtain

$$
\begin{aligned}
f(\bar{H} \bar{K}) & =f(|H \cap K| \overline{H K})=|H \cap K| f(\overline{H K}) \\
& =|H \cap K| \overline{\beta(H K)}=|H \cap K| \overline{\beta(H) \beta(K)} \\
& =|\beta(H) \cap \beta(K)| \overline{\beta(H) \beta(K)}=\overline{\beta(H)} \overline{\beta(K)}=f(\bar{H}) f(\bar{K}),
\end{aligned}
$$

so $f$ preserves the ordinary product, which proves $f \in \operatorname{Aut}(F \overline{\mathcal{L}})$.

Now we only need to show that $\chi$ is surjective. Suppose $\gamma$ is any S-ring automorphism of $F \overline{\mathcal{L}}$. For any $H \in \mathcal{L}$, we have $\gamma(\bar{H})=\bar{K}$ for some subgroup $K \leq G$ by Theorem 1.5(ii). Since $F \overline{\mathcal{L}}$ is a rational S-ring, $K$ is necessarily characteristic. By the linear independence of characteristic subgroups (Corollary [3.13), we must have $K \in \mathcal{L}$. Thus $\gamma$ permutes the basis elements $\{\bar{H}: H \in \mathcal{L}\}$ of $F \overline{\mathcal{L}}$. We can then define a bijection $\beta: \mathcal{L} \rightarrow \mathcal{L}$ by setting $\beta(H)=K$ where $K$ is the subgroup of $G$ such that $\gamma(\bar{H})=\bar{K}$, so that $\overline{\beta(H)}=\gamma(\bar{H})$ for all $H \in \mathcal{L}$. Once we show that $\beta$ is a lattice automorphism of $\mathcal{L}$, we will have $\gamma=\chi(\beta)$, which will complete the proof.

Theorem 2.3 implies

$$
\begin{aligned}
\overline{\beta(H \cap K)} & =\gamma(\overline{H \cap K})=\gamma(\bar{H} \circ \bar{K}) \\
& =\gamma(\bar{H}) \circ \gamma(\bar{K})=\overline{\beta(H)} \circ \overline{\beta(K)}=\overline{\beta(H) \cap \beta(K)}
\end{aligned}
$$


so that $\beta(H \cap K)=\beta(H) \cap \beta(K)$. Observe that $|\beta(H)|=|H|$ by Theorem 1.5(i), hence

$$
|H \cap K|=|\beta(H \cap K)|=|\beta(H) \cap \beta(K)|,
$$

so a further application of Theorem 2.3 implies

$$
\begin{aligned}
\overline{\beta(H K)} & =\gamma(\overline{H K})=\gamma\left(\frac{1}{|H \cap K|} \bar{H} \bar{K}\right)=\frac{1}{|H \cap K|} \gamma(\bar{H}) \gamma(\bar{K}) \\
& =\frac{1}{|H \cap K|} \overline{\beta(H)} \overline{\beta(K)}=\frac{1}{|\beta(H) \cap \beta(K)|} \overline{\beta(H)} \overline{\beta(K)}=\overline{\beta(H) \beta(K)}
\end{aligned}
$$

so that $\beta(H K)=\beta(H) \beta(K)$. This proves that $\beta$ is a lattice automorphism of $\mathcal{L}$, as desired.

\section{Automorphisms of S-rings over cyclic groups}

In this section, we again take all S-rings over a coefficient field $F$ of characteristic zero.

In [14], Leung and Man give a recursive classification of all S-rings over cyclic groups. We give a brief description of this classification. They give three basic methods of constructing S-rings over a group $G$ :

(I) Given a subgroup $\Omega \leq \operatorname{Aut}(G)$, let $T_{1}, \ldots, T_{n}$ be the orbits of $\Omega$ acting on $G$. Then $T_{1}, \ldots, T_{n}$ form a Schur partition of $G$.

(II) Suppose $G=H \times K$ for nontrivial subgroups $H, K \leq G$, and suppose $S_{H}$ is an S-ring over $H$ with basic sets $C_{1}, \ldots, C_{h}$ and $S_{K}$ is an S-ring over $K$ with basic sets $D_{1}, \ldots, D_{k}$. Then the product sets $C_{i} D_{j}, 1 \leq i \leq h, 1 \leq j \leq k$, form a Schur partition of $G$.

(III) Suppose $H$ and $K$ are nontrivial, proper subgroups of $G$ with $H \leq K$ and $H \unlhd G$, and let $S_{K}$ be an S-ring over $K$ with basic sets $C_{1}, \ldots, C_{k}$ and $S_{G / H}$ be an S-ring over $G / H$ with basic sets $D_{1}, \ldots, D_{k}$, and suppose that $\pi\left(S_{K}\right)=$ $F(K / H) \cap S_{G / H}$, where $\pi: G \rightarrow G / H$ is the natural projection map, extended to a natural projection map of the group algebra $F G$ onto $F(G / H)$. Then

$$
G=C_{1} \cup \cdots \cup C_{k} \cup\left\{\pi^{-1}\left(D_{i}\right): i \in\{1, \ldots, k\}, D_{i} \nsubseteq K / H\right\}
$$

forms a Schur partition of $G$.

In the case of cyclic groups, the S-ring constructed in (I) is called a cyclotomic S-ring (a more general definition of cyclotomic S-rings, together with some of their applications, is found in [17]). The S-ring constructed in (II) is denoted $S_{H} \cdot S_{K}$ and is called the dot product (also denoted $S_{H} \otimes S_{K}$ and called the tensor product) of $S_{H}$ and $S_{K}$. The S-ring constructed in (III) is denoted $S_{K} \wedge S_{G / H}$ and is called the wedge product (or generalized wreath product) of $S_{K}$ and $S_{G / H}$.

The main theorem of Leung and Man ([14, Theorem 3.7]) may then be stated as follows: 
Theorem 5.1. Every nontrivial S-ring over a cyclic group $G$ is either cyclotomic, a dot product, or a wedge product.

Note that the constructions (I), (II), and (III) can be used to produce S-rings over an arbitrary group $G$, but if $G$ is not cyclic then it is not necessarily true that all S-rings can be constructed using these methods. For instance, over elementary abelian $p$-groups we have the following (for proofs, see [12, Theorem 8.4, Example 8.5]):

Theorem 5.2. Let $p$ be a prime with $p \geq 5$. Then there are $S$-rings over $Z_{p} \times Z_{p}$ which cannot be constructed as in (I), (II), or (III).

Theorem 5.3. There are $S$-rings over $Z_{3} \times Z_{3} \times Z_{3} \times Z_{3}$ which cannot be constructed as in (I), (II), or (III).

In contrast, all S-rings over $Z_{3} \times Z_{3}$ and $Z_{3} \times Z_{3} \times Z_{3}$ can be constructed as in (I). In [12, Question 8.6], the question was asked of whether all S-rings over an elementary abelian 2-group can be constructed as in (I). We can now give a negative answer to this:

Theorem 5.4. There are S-rings over $Z_{2}^{6}$ which cannot be constructed as in (I), (II), or (III).

Proof. Consider $G=Z_{2}^{6}$ as the additive group of the finite field $F_{64}$. Since $x^{6}+x^{4}+$ $x^{3}+x+1$ is primitive over $\mathbb{F}_{2}, F_{64}^{\times}$has a generator $\omega$ with $\omega^{6}+\omega^{4}+\omega^{3}+\omega+1=0$. The action of multiplication by $\left\langle\omega^{9}\right\rangle$ partitions $F_{64}^{\times}$into 9 orbits (each of size 7 ),

$$
C_{i}=\left\{\omega^{i+9 j}: j \in\{0 . .6\}\right\}, \quad i=0, \ldots, 8 .
$$

We have found using MAGMA 4 that

$$
\{0\}, C_{0} \cup C_{1} \cup C_{2} \cup C_{3} \cup C_{4}, C_{5} \cup C_{6} \cup C_{7} \cup C_{8}
$$

forms a Schur partition of $G$ whose corresponding S-ring $S$ cannot be constructed as in (I), (II), or (III) (For (II) and (III), this is fairly obvious: since basic sets have size 35,28 , and 1 , it is clear that $S$ has no nontrivial proper $S$-subgroup, i.e., $S$ is a primitive S-ring.)

Remark. An exhaustive enumeration using MAGMA shows that all S-rings over $Z_{2}^{n}$ for $n \leq 4$ can be constructed as in (I). The question in the case $n=5$ remains open; this remaining case would probably still be feasible to answer by exhaustive enumeration, if one reduced the computation by taking advantage of the symmetry of the group.

We now turn to the main result of this section:

Theorem 5.5. If $S$ is an $S$-ring over a cyclic group $Z_{n}$, then $\operatorname{Aut}(S)$ is abelian. 
Before proving this, we need a few elementary lemmas, proofs of which may be found in [12, Lemmas 8.8, 8.9, 8.10]. A version of Lemma 5.6 can be found in [23, Theorem 23.9], while more general versions of Lemmas 5.7 and 5.8 can be found in [19, Propositions 3.1, 3.4].

Lemma 5.6 (Wielandt). Suppose $S$ is an $S$-ring over an abelian group $G$ and $m$ is an integer relatively prime to $|G|$. If $\bar{C} \in S$, then also $\bar{C}^{(m)} \in S$. Moreover, if $\bar{C}$ is a basic element of $S$ then $\bar{C}^{(m)}$ is also a basic element of $S$.

Lemma 5.7. Suppose $S$ is an $S$-ring over an abelian group $G$ and $\phi \in \operatorname{Aut}(S)$. Then for any simple quantity $\bar{C} \in S$ and any integer $m$ relatively prime to $|G|$, we have $\phi\left(C^{(m)}\right)=\phi(C)^{(m)}$.

Lemma 5.8. Suppose $S$ is a cyclotomic $S$-ring over $Z_{n}$. Let $\phi$ be an automorphism of $S$. Then for any basic set $T$ of $S$, there is an integer $m$ relatively prime to $n$ such that $\phi(T)=T^{(m)}$.

Remark. Lemma 5.6 says that over an abelian group, every Cayley isomorphism is actually an automorphism of the S-ring. Lemma 5.7 says that the Cayley automorphisms are in the center of the automorphism group of the S-ring. Finally, Lemma 5.8 says that for a cyclotomic S-ring over a cyclic group, every S-ring automorphism "locally" behaves like a Cayley automorphism. (In Lemma 5.8, the assumption that the S-ring be cyclotomic is actually unnecessary. However, the generalization omitting this hypothesis is a much deeper result which we will not need to use directly; it may be found in [19, Theorem 1.1'], where it plays a key role in the proof of Muzychuk's result cited in Theorem 6.1.)

Proof of Theorem 5.5. If $S$ is a trivial S-ring, then $\operatorname{Aut}(S)$ is also trivial, hence abelian, and we are done. So first consider the case that $S$ is cyclotomic. Each basic set of $S$ then consists of elements of the same order. Let $\mathcal{T}_{d}$ be the collection of basic sets of $S$ containing elements of order $d$. We can consider $\operatorname{Aut}(S)$ as a permutation group acting on the basic sets of $S$, and by Lemma $[5.8$, for each $d, \operatorname{Aut}(S)$ permutes the basic sets of $\mathcal{T}_{d}$ among themselves. If we let $A_{d}$ denote the restriction of $\operatorname{Aut}(S)$ to $\mathcal{T}_{d}$, then $\operatorname{Aut}(S)$ is a subdirect product of all the $A_{d}$ 's, so it suffices to show that each $A_{d}$ is abelian. Fix a divisor $d$ of $n$, and let $T$ be a basic set in $\mathcal{T}_{d}$. For any $k$ relatively prime to $n, T^{(k)}$ is another basic set of $\mathcal{T}_{d}$ by Lemma 5.6, and every basic set of $\mathcal{T}_{d}$ has this form, for if $T^{\prime}$ is any basic set in $\mathcal{T}_{d}$, there is an integer $l$ relatively prime to $n$ such that $T^{(l)} \cap T^{\prime} \neq \emptyset$, hence $T^{(l)}=T^{\prime}$. Now, by Lemma 5.8, $\phi(T)=T^{(m)}$ for some $m$ relatively prime to $n$. It follows by Lemma 5.7 that for any basic set $T^{(k)} \in \mathcal{T}_{d}$,

$$
\phi\left(T^{(k)}\right)=\phi(T)^{(k)}=\left(T^{(m)}\right)^{(k)}=\left(T^{(k)}\right)^{(m)} .
$$

Thus $\phi\left(T^{\prime}\right)=\left(T^{\prime}\right)^{(m)}$ for any basic set $T^{\prime} \in \mathcal{T}_{d}$. From this it is evident that $A_{d}$ is abelian.

Now suppose $S$ is a dot product $S_{H} \cdot S_{K}$. By induction we may assume $\operatorname{Aut}\left(S_{H}\right)$ and $\operatorname{Aut}\left(S_{K}\right)$ are abelian. Let $\phi$ be an element of $\operatorname{Aut}(S)$. Since $H$ is the unique subgroup 
of order $|H|$ in $G$, Theorem 1.5(i,ii) implies $\phi(\bar{H})=\bar{H}$, and similarly $\phi(\bar{K})=\bar{K}$. So $\phi\left(S_{H}\right)$ is an S-ring over $H$ which is isomorphic to $S_{H}$. By the result of Muzychuk cited in Theorem 6.1, the only such S-ring is $S_{H}$ itself, so we must have $\phi\left(S_{H}\right)=S_{H}$ and likewise $\phi\left(S_{K}\right)=S_{K}$. So $\left.\phi\right|_{S_{H}} \in \operatorname{Aut}\left(S_{H}\right)$ and $\left.\phi\right|_{S_{K}} \in \operatorname{Aut}\left(S_{K}\right)$. Given another automorphism $\psi \in \operatorname{Aut}(S)$, and any basic set $C D$ of $S$, where $C$ is a basic set of $S_{H}$ and $D$ is a basic set of $S_{K}$, we have $\phi(\psi(\bar{C}))=\psi(\phi(\bar{C}))$ and $\phi(\psi(\bar{D}))=\psi(\phi(\bar{D}))$ since $\operatorname{Aut}\left(S_{H}\right)$ and $\operatorname{Aut}\left(S_{K}\right)$ are abelian, hence

$$
\begin{aligned}
\phi(\psi(\overline{C D})) & =\phi(\psi(\bar{C} \bar{D}))=\phi(\psi(\bar{C}) \psi(\bar{D}))=\phi(\psi(\bar{C})) \phi(\psi(\bar{D})) \\
& =\psi(\phi(\bar{C})) \psi(\phi(\bar{D}))=\psi(\phi(\bar{C}) \phi(\bar{D}))=\psi(\phi(\bar{C} \bar{D}))=\psi(\phi(\overline{C D}))
\end{aligned}
$$

which proves that $\phi$ and $\psi$ commute, so $\operatorname{Aut}(S)$ is abelian.

Finally suppose $S$ is a wedge product $S_{K} \wedge S_{G / H}$. As above, given an automorphism $\phi \in \operatorname{Aut}(S)$, we have $\left.\phi\right|_{S_{K}} \in \operatorname{Aut}\left(S_{K}\right)$. Now define $F$-linear maps $\pi: F G \rightarrow F(G / H)$ and $\pi^{\prime}: F(G / H) \rightarrow F G$ by

$$
\begin{aligned}
& \pi: g \mapsto g H \\
& \pi^{\prime}: g H \mapsto g \bar{H} .
\end{aligned}
$$

The following relations are easily checked:

$$
\begin{aligned}
\pi\left(\pi^{\prime}(x)\right) & =|H| x \\
\pi(x y) & =\pi(x) \pi(y) \\
\pi^{\prime}(x y) & =\frac{1}{|H|} \pi^{\prime}(x) \pi^{\prime}(y)
\end{aligned}
$$

Consider the linear map $\phi^{*}: S_{G / H} \rightarrow S_{G / H}$ given by the composite function $\phi^{*}=$ $\frac{1}{|H|} \pi \phi \pi^{\prime}$. We need to justify that this is well-defined. Note that if $B$ is a basic set of $S_{G / H}$ then $\pi^{\prime}(\bar{B})=\bar{C}$ where $C$ is a basic set of $S_{G}$ and $C$ is a union of cosets of $H$, so that $\bar{C} \bar{H}=|H| \bar{C}$. Applying $\phi$ to boths sides gives $\phi(\bar{C}) \bar{H}=|H| \phi(\bar{C})$, so that the basic set $D=\phi(C)$ of $S_{G}$ is also union of cosets of $H$. Then $\pi(\bar{D})=|H| \bar{E}$ for a basic set $E$ of $S_{G / H}$. Putting this together, we have

$$
\phi^{*}(\bar{B})=\frac{1}{|H|} \pi\left(\phi\left(\pi^{\prime}(\bar{B})\right)\right)=\frac{1}{|H|} \pi(\phi(\bar{C}))=\frac{1}{|H|} \pi(\bar{D})=\bar{E},
$$

so that $\phi^{*}$ is well-defined and maps basic quantities to basic quantities. Now, we have

$$
\left(\frac{1}{|H|} \pi^{\prime} \phi^{-1} \pi\right) \phi^{*}=\frac{1}{|H|^{2}} \pi^{\prime} \phi^{-1} \pi \pi^{\prime} \phi \pi=\frac{1}{|H|} \pi^{\prime} \phi^{-1} \phi \pi=\frac{1}{|H|} \pi^{\prime} \pi=1_{F G},
$$

so $\phi^{*}$ is a bijection. Finally,

$$
\begin{aligned}
\phi^{*}(x y) & =\frac{1}{|H|}\left(\pi \phi \pi^{\prime}\right)(x y)=\frac{1}{|H|^{2}} \pi\left(\phi\left(\pi^{\prime}(x) \pi^{\prime}(y)\right)\right) \\
& =\frac{1}{|H|^{2}} \pi\left(\phi\left(\pi^{\prime}(x)\right)\right) \pi\left(\phi\left(\pi^{\prime}(y)\right)\right)=\phi^{*}(x) \phi^{*}(y)
\end{aligned}
$$


so $\phi^{*}$ is an S-ring automorphism of $S_{G / H}$.

By induction we may assume $\operatorname{Aut}\left(S_{K}\right)$ and $\operatorname{Aut}\left(S_{G / H}\right)$ are abelian. Then, given another automorphism $\psi \in \operatorname{Aut}(S)$ and a basic set $C$ of $S$, to complete the proof, it suffices to show $\phi(\psi(\bar{C}))=\psi(\phi(\bar{C}))$. We have two cases: If $C \subseteq K$, then

$$
\phi(\psi(\bar{C}))=\left.\phi\right|_{S_{K}}\left(\left.\psi\right|_{S_{K}}(\bar{C})\right)=\left.\psi\right|_{S_{K}}\left(\left.\phi\right|_{S_{K}}(\bar{C})\right)=\psi(\phi(\bar{C}))
$$

and we are done. Suppose instead that $C \nsubseteq K$. Then $C$ is a union of cosets of $H$, hence $\pi^{\prime}(\pi(\bar{C}))=|H| \bar{C}$. As above, $\phi(C)$ is also a union of cosets of $H$. Similarly, so are $\psi(C), \phi(\psi(C))$, and $\psi(\phi(C))$. It follows that

$$
\left(\pi^{\prime} \pi \phi\right)(\bar{C})=\pi^{\prime}(\pi(\overline{\phi(C)}))=|H| \overline{\phi(C)}=|H| \phi(\bar{C})
$$

and likewise

$$
\begin{aligned}
\left(\pi^{\prime} \pi \psi\right)(\bar{C}) & =|H| \psi(\bar{C}) \\
\left(\pi^{\prime} \pi \phi \psi\right)(\bar{C}) & =|H|(\phi \psi)(\bar{C}) \\
\left(\pi^{\prime} \pi \psi \phi\right)(\bar{C}) & =|H|(\psi \phi)(\bar{C})
\end{aligned}
$$

From all of this it follows that

$$
\begin{aligned}
(\phi \psi)(\bar{C}) & =\frac{1}{|H|}\left(\pi^{\prime} \pi \phi \psi\right)(\bar{C})=\frac{1}{|H|^{2}}\left(\pi^{\prime} \pi \phi \pi^{\prime} \pi \psi\right)(\bar{C}) \\
& =\frac{1}{|H|^{3}}\left(\pi^{\prime} \pi \phi \pi^{\prime} \pi \psi \pi^{\prime} \pi\right)(\bar{C}) \\
& =\frac{1}{|H|}\left(\pi^{\prime} \phi^{*} \psi^{*} \pi\right)(\bar{C})=\frac{1}{|H|}\left(\pi^{\prime} \psi^{*} \phi^{*} \pi\right)(\bar{C}) \\
& =\frac{1}{|H|^{3}}\left(\pi^{\prime} \pi \psi \pi^{\prime} \pi \phi \pi^{\prime} \pi\right)(\bar{C}) \\
& =\frac{1}{|H|^{2}}\left(\pi^{\prime} \pi \psi \pi^{\prime} \pi \phi\right)(\bar{C})=\frac{1}{|H|}\left(\pi^{\prime} \pi \psi \phi\right)(\bar{C})=(\psi \phi)(\bar{C}),
\end{aligned}
$$

so that $\phi$ and $\psi$ commute, as desired.

We observe that Theorem 5.5 is false if the coefficient field $F$, or more generally the coefficient ring $R$, has nonzero characteristic:

Example 5.9. Let $R$ have characteristic $n>0$. Set $G=Z_{4 n}=\langle t\rangle$ and $H=Z_{n} \leq G$. Define $S_{H}$ to be the S-ring $S=S_{H} \wedge S_{G / H}$ where $S_{H}$ is the trivial S-ring over $H$ and $S_{G / H}$ is the full group algebra $R(G / H)$. Then $S$ has five basic sets

$$
\{1\}, Z_{n}-\{1\}, T_{1}, T_{2}, T_{3}
$$

where $T_{i}=t^{i} Z_{n}$. Then in $R G$ we have $\bar{T}_{i} \bar{T}_{j}=n t^{i+j} \bar{Z}_{n}=0$ for all $i, j \in\{1,2,3\}$ while $\left(\bar{Z}_{n}-1\right) \bar{T}_{i}=-\bar{T}_{i}$. Thus $\operatorname{Aut}(S) \cong \mathrm{Sym}_{3}$ is non-abelian. Over a ring with characteristic zero, this same Schur partition gives an S-ring with automorphism group isomorphic to $Z_{2}$. 
We observe that the Leung-Man classification of S-rings over cyclic groups does not hold if the coefficient field has nonzero characteristic (see, e.g., [12, Example 1.11]), but Example 5.9 shows that this is not the only reason that Theorem 5.5 fails in this case. This suggests two problems, both of which we can expect to be difficult:

Problem 5.10. Classify the S-rings over cyclic groups for coefficient rings of nonzero characteristic.

Problem 5.11. Describe the automorphism groups of such S-rings.

\section{Converse to Muzychuk's Theorem}

A remarkable theorem of Muzychuk states:

Theorem 6.1 ([19]). Two S-rings over a cyclic group $Z_{n}$ are isomorphic if and only if they are identical.

We prove a converse to this result. But first we need the following lemma:

Lemma 6.2. Let $G$ be a finite group which is not cyclic. Then $G$ has a subgroup which is not characteristic.

Proof. By way of contradiction, suppose every subgroup of $G$ is characteristic. Then in particular every subgroup of $G$ is normal. If $G$ is non-abelian, then $G$ is a Hamiltonian group (i.e., a nonabelian group in which every subgroup is normal) and we may write $G=Q \times A$ where $Q$ is an 8-element quaternion group $\langle i, j\rangle$ and $A$ is abelian [21, 9.7.4]. But in this case $\langle i\rangle$ is a subgroup of $G$ which is not characteristic, since there is an automorphism of $Q$ mapping $\langle i\rangle$ to $\langle j\rangle$ and this automorphism extends to an automorphism of $G$. Therefore $G$ must be abelian.

Since $G$ is not cyclic, some Sylow $p$-subgroup of $G$ is not cyclic and, by the Fundamental Theorem of finitely-generated abelian groups, we may write $G=\langle t\rangle \times$ $\langle s\rangle \times A$ where $|t|=p^{a}$ and $|s|=p^{b}$ for some $a$ and $b$ where $1 \leq a \leq b$. Then $\langle s\rangle$ is not characteristic, since an automorphism $\phi$ is determined by setting $\phi(s)=t s, \phi(t)=t$, and $\phi(a)=a$ for all $a \in A$.

We remark that, by a similar method of proof, Lemma 6.2 may be extended to infinite non-abelian groups and to finitely generated abelian groups. However, there are non-cyclic infinitely generated abelian groups in which every subgroup is characteristic, an example being the direct sum $\sum_{p \text { prime }} Z_{p}$.

Theorem 6.3. Let $G$ be a finite group which is not cyclic. Then there exist distinct Cayley-isomorphic S-rings $S_{1}$ and $S_{2}$ over $G$.

Proof. By Lemma 6.2, let $H$ be a subgroup of $G$ which is not characteristic. Choose some $\phi \in \operatorname{Aut}(G)$ such that $\phi(H) \neq H$. Then $S_{1}=F\{1, \bar{H}, \bar{G}\}$ and $S_{2}=F\{1, \overline{\phi(H)}, \bar{G}\}$ are S-rings over $G$ which are Cayley-isomorphic. We only need to verify that they are distinct. The basic quantities of $S_{1}$ are $\{1, \bar{H}-1, \bar{G}-\bar{H}\}$ while the basic quantities of 
$S_{2}$ are $\{1, \overline{\phi(H)}-1, \bar{G}-\overline{\phi(H)}\}$. If $S_{1}=S_{2}$ then the basic quantities of the two S-rings must be the same (in some order), so either $\bar{H}-1=\overline{\phi(H)}-1$ or $\bar{H}-1=\bar{G}-\overline{\phi(H)}$. The former is impossible since $H \neq \phi(H)$. The latter would imply $G=H \cup \phi(H)$, which is impossible, since no group is the union of two proper subgroups.

\section{Some examples}

Example 7.1. Let $G=Z_{p} \times Z_{p^{3}}$ for an odd prime $p$. Let $H_{1}, \ldots, H_{6}$ be the characteristic subgroups of $G$, in the order shown in Table 1. Using Theorem 1.1, it is easy to check that $S=F\left\{1, \bar{H}_{2}, \bar{H}_{3}+\bar{H}_{4}, \bar{H}_{5}, \bar{G}\right\}$ is a rational S-ring. We show that $S$ cannot be constructed as in Theorem 2.3. Suppose $S=F \overline{\mathcal{L}}$ for some lattice $\mathcal{L}$. By Corollary 3.13 , the elements $\{\bar{H}: H \in \mathcal{L}\}$ are linearly independent, hence form a basis for $S$. So $\operatorname{dim} S=|\mathcal{L}|$. Now $\operatorname{dim} S=5$, yet, by applying Corollary 3.13 again, it is easy to see that $1, H_{2}, H_{5}$, and $G$ are the only four subgroups of $G$ which are $S$-sets, hence $|\mathcal{L}| \leq 4$, a contradiction.

Over other abelian $p$-groups, it is easy to construct many rational S-rings similar to Example 7.1, where the basic quantities are sums of characteristic subgroups, chosen in such a way as to ensure closure under the Hadamard and ordinary product; but, thus far, it has not been possible to extend this construction to give a complete classification of rational S-rings over abelian $p$-groups. Some of the difficulty is indicated by the following example:

Example 7.2. Let $G=Z_{p} \times Z_{p^{3}} \times Z_{p^{5}}$ where $p$ is any prime. Let $H_{1}, \ldots, H_{18}$ be the regular characteristic subgroups of $G$, as in Table 2. By direct computation, one can show using Corollary 1.2 that

$$
S=F\left\{1, \bar{H}_{5}, \bar{H}_{6}+\bar{H}_{7}-\bar{H}_{8}-\bar{H}_{11}, \bar{H}_{8}+3 \bar{H}_{11}-\bar{H}_{12}-\bar{H}_{13}, \bar{H}_{14}, \bar{G}\right\}
$$

is an S-ring over $G$ if and only if $p=3$. It is of course also possible to present $S$ in terms of its basic elements:

$S=F\left\{1, \bar{O}_{2}+\bar{O}_{3}+\bar{O}_{4}+\bar{O}_{5}, \bar{O}_{6}+\bar{O}_{7}+\bar{O}_{14}, \bar{O}_{12}+\bar{O}_{13}, \bar{O}_{8}+\bar{O}_{10}+\bar{O}_{11}, \bar{O}_{9}+\bar{O}_{15}+\bar{O}_{16}+\bar{O}_{17}+\bar{O}_{18}\right\}$

where $O_{i}=O(\mathbf{a})$ for $H_{i}=R(\mathbf{a})$. This S-ring does not have a basis consisting of sums of characteristic subgroups. This example also shows that choice of prime $p$ can make a difference in determining whether a partition of automorphism classes of $G$ is a Schur partition or not, and that it is not merely a question of whether $p=2$. For a further discussion of this and related examples, see [12, Example 5.28] (But note that the initial basis given there for $S$ is erroneous; we have given a correct basis above).

Such examples lead us to a conjecture:

Conjecture 7.3. Let $\left(\lambda_{1}, \ldots, \lambda_{n}\right)$ be a tuple of integers, $1 \leq \lambda_{1} \leq \cdots \leq \lambda_{n}$. Then, as $p \rightarrow \infty$, the set of Schur partitions of the abelian p-group

$$
G=Z_{p^{\lambda_{1}}} \times \cdots \times Z_{p^{\lambda_{n}}}
$$


is eventually constant, and for sufficiently large p, every rational S-ring over $G$ has a basis consisting of sums of characteristic subgroups (where, here we are abusing notation slightly by thinking of the Schur partitions as partitions of $\mathcal{C}(G)$, rather than of $G$ itself).

One natural approach to classifying rational S-rings over abelian $p$-groups would involve (1) answering this conjecture, (2) giving an explicit description of which sums of characteristic subroups are allowed, and (3) attempting to understand the apparently "exceptional" rational S-rings which occur when $p$ is small relative to $n$.

\section{References}

[1] Reinhold Baer. Types of elements and characteristic subgroups of abelian groups. Proc. London Math. Soc., 39(2054):481-514, 1934.

[2] Garrett Birkhoff. Subgroups of abelian groups. Proc. London Math. Soc., 38:385401, 1935.

[3] Garrett Birkhoff. Sobre los grupos de automorfismos. Revista de la Unión Matemática Argentina, 11:155-157, 1946.

[4] Wieb Bosma, John Cannon, and Catherine Playoust. The Magma algebra system. J. Symbolic Comput., 24(3-4):235-265, 1997.

[5] Peter Crawley and Robert P. Dilworth. Algebraic Theory of Lattices. PrenticeHall: Englewood Cliffs, New Jersey, 1973.

[6] B. A. Davey and H. A. Priestley. Introduction to Lattices and Order, 2nd Ed. Cambridge University Press, 2002.

[7] S. Foldes. On automorphism groups of graphs and distributive lattices. Algebra Universalis, 41:115-120, 1999.

[8] G. Grätzer, H. Lakser, and E. T. Schmidt. On a result of Birkhoff. Periodica Mathematica Hungarica, 30(3):183-188, 1995.

[9] G. Grätzer, E. T. Schmidt, and D. Wang. A short proof of a theorem of Birkhoff. Algebra Universalis, 37(2):253-255, 1997.

[10] H. Hermes. Einführung in die Verbandstheorie. Springer-Verlag, 1955.

[11] Brent Kerby and Emma Turner. Characteristic subgroups of finite abelian groups, preprint. http://arxiv.org/abs/0905.1885. 2008.

[12] Brent L. Kerby. Rational schur rings over abelian groups. Master's thesis, Brigham Young University. http://etd.byu.edu, 2008.

[13] K. H. Leung and S. H. Man. On schur rings over cyclic groups. Israel J. Math., 106:251-267, 1998 
[14] Ka Hin Leung and Shing Hing Man. On Schur rings over cyclic groups, II. Journal of Algebra, 183:273-285, 1996.

[15] G. A. Miller. Determination of all the characteristic subgroups of any abelian group. American Journal of Mathematics, 27(2):15-24, 1905.

[16] G. A. Miller, H. F. Blichfeldt, and L. E. Dickson. Theory and Applications of Finite Groups. John Wiley and Sons, Inc., 1916.

[17] Mikhail Muzychuk and Ilia Ponomarenko. Schur rings. European Journal of Combinatorics, 2009, doi: 10.1016/j.ejc.2008.11.006.

[18] Mikhail E. Muzychuk. The structure of rational Schur rings over cyclic groups. European Journal of Combinatorics, 14:479-490, 1993.

[19] Mikhail E. Muzychuk. On the structure of basic sets of Schur rings over cyclic groups. Journal of Algebra, 169:655-678, 1994.

[20] Issai Schur. Zur Theorie der einfach transitiven Permutationsgruppen. Sitzungsber. Preuss. Akad. Wiss., Phys.-Math., pages 598-623, 1933.

[21] W. R. Scott. Group Theory. Englewood Cliffs, New Jersey: Prentice-Hall, 1964.

[22] H. de Vries and A. B. de Miranda. Groups with a small number of automorphisms. Mathematische Zeitschrift, 68:450-464, 1957.

[23] Helmut Wielandt. Finite Permutation Groups. New York, Academic Press, 1964. 